\title{
CEA/Tetanus Toxoid T Helper Epitope Fusion Protein-Expressing DNA Plasmid Vaccine
}

National Cancer Institute

\section{Source}

National Cancer Institute. CEAVT etanus Toxoid T Helper Epitope Fusion Protein-

Expressing DNA Plasmid Vaccine. NCI Thesaurus. Code C90555.

A plasmid vaccine encoding wild type human carcinoembryonic antigen (CEA) fused to a tetanus toxoid T helper epitope, with potential antineoplastic activity. Upon vaccination and subsequent intradermal electroporation, CEA/tetanus toxoid T helper epitope fusion protein-expressing DNA plasmid vaccine may stimulate the immune system to mount a cytotoxic T-lymphocyte $(C T L)$ response against CEA-expressing tumor cells. CEA, a tumor associated antigen, is overexpressed in a variety of cancer cell types. The tetanus toxoid helper peptide epitope, obtained from the bacterial Clostridium tetani toxoid, binds to class II MHC molecules and increases the helper T-cell response thereby inducing an increased and long-term immune response. 